\title{
Avaliação dos parâmetros tempo, corrente e pressão na soldagem por resistência elétrica de compósitos PEl/fibras contínuas: influência na resistência mecânica.
}

\author{
Ana Beatriz Ramos Moreira Abrahão ${ }^{1}$, Jonas Frank Reis², \\ Samia Danuta Brejão ${ }^{1}$, Vinícius Garcia Ribeiro ${ }^{2}$, \\ Michelle Leali Costa ${ }^{1}$, Edson Cocchieri Botelho ${ }^{1}$
}

\author{
${ }^{1}$ Departamento de Materiais e Tecnologia - FEG/UNESP, Guaratinguetá, São Paulo, SP \\ email: ana.abrahao@fatec.sp.gov.br \\ ${ }^{2}$ Faculdade de Tecnologia de Pindamonhangaba-FATEC, Pindamonhangaba, São Paulo, SP \\ e-mail: jonasfrankreis@gmail.com
}

\begin{abstract}
RESUMO
Atualmente, muitos componentes destinados à indústria aeronáutica, que anteriormente utilizavam compósitos com matriz termorrígida, vêm sendo substituídos por aqueles que apresentam matriz termoplástica, devido principalmente às melhores propriedades mecânicas obtidas, possibilidade de reaproveitamento do mesmo e a facilidade na fabricação de peças grandes e complexas, que serão integradas para formar o componente final desejado, assim como a sua temperatura de serviço mais elevada. Entretanto, um dos principais problemas da utilização de compósitos poliméricos em aplicações estruturais consiste em sua união efetiva para a integração de componentes. Nos últimos anos, a soldagem por resistência elétrica vem sendo considerada como uma das mais promissoras técnicas para a união de compósitos, pois se trata de um processo com pouca preparação de superfície e com qualidade aceitável no setor industrial. O principal objetivo deste trabalho consiste no estudo de parâmetros de soldagem por resistência elétrica para o compósito polimérico PEI poli (éter-imida) reforçado com fibras de carbono e de vidro (sistema híbrido) para aplicações aeronáuticas. As amostras do compósito foram soldadas e submetidas ao ensaio de lap shear para obtenção de tensão de ruptura ao cisalhamento de juntas simples. A partir deste ensaio foram estabelecidas as variáveis mais adequadas para utilização neste processo. O método utilizado foi um planejamento experimental fatorial completo que permitiu, com um número menor de experimentos, explorar todo o espaço experimental de interesse, estabelecendo os valores mais adequados de corrente elétrica, pressão e tempo para o processo de soldagem estudado.
\end{abstract}

Palavras-chave: Compósitos, Soldagem por Resistência Elétrica, PEI, Planejamento Experimental.

\begin{abstract}
Nowadays, many components applied in the aerospace industry, which had been previously used with thermoset matrix composites, are being replaced by those with thermoplastic matrix, which is mainly due to having better mechanical properties, the possibility of reuse and ease in manufacturing large and complex parts which will be integrated to form the desired component with higher service temperature. However, a major problem concerning the use of polymer composites for structural applications consists in its effective integration of components. Electrical resistance welding has been considered as one of the most promising techniques for bonding thermoplastic composites because it is a quick process with little surface preparation. The main objective of this work is to study the resistance welding parameters for PEI (polyether imide), and carbon and glass (hybrid) fibers laminates for aeronautical applications. The composite specimens were welded and subjected to the lap shear test in order to establish the most suitable variable in this process. It was used a full factorial design that allowed, with fewer experiments, exploring the whole experimental area of interest, establishing values of electric current, pressure and time for the studied welding process.
\end{abstract}

Keywords: Composites, Electrical Resistance Welding, PEI, Experimental Design 


\section{INTRODUÇÃO}

Desde o advento da corrida espacial, constantes desenvolvimentos vêm sendo realizados em compósitos estruturais visando aplicações aeroespaciais. Devido à força superior e a relação rigidez-peso, materiais compósitos estão encontrando grandes aplicações de alta tecnologia na indústria aeronáutica [1].

Um exemplo que pode ser associado à utilização destes materiais é o Boeing 787 Dreamliner que foi a primeira aeronave comercial a ser fabricada com mais de 50\% de material compósito em sua estrutura (porcentagem em massa). A Figura 1 apresenta uma ilustração indicando os principais materiais utilizados nas estruturas do Boeing 787 Dreamliner [2].

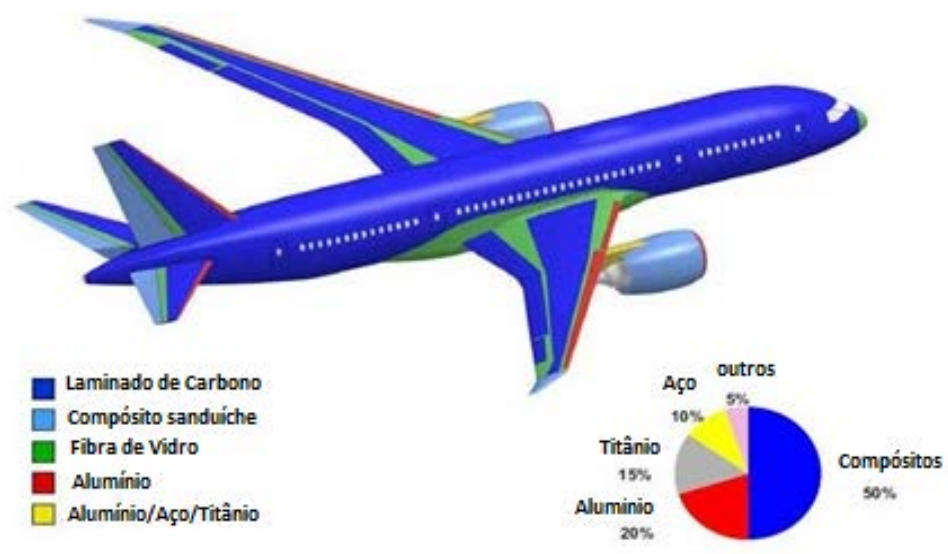

Figura 1: Os principais materiais da estrutura da aeronave Boeing 787 [2].

Compósitos termoplásticos oferecem várias vantagens de processamento e desempenho mecânico quando comparados aos termorrígidos. Os materiais compósitos termoplásticos apresentam elevada tolerância a danos, excelente resistência à corrosão, alta resistência à ruptura, boas resistências ao impacto e à fadiga, baixos custos de armazenamento e vida útil de prateleira infinita. No setor aeronáutico, o uso de compósitos termoplásticos é promissor na construção de fuselagens, permitindo com isto uma redução de peso em torno de $25 \%$, em relação às estruturas metálicas utilizadas. Adicionalmente, os compósitos termoplásticos têm continuamente se mantidos como uma alternativa de aplicação em estruturas, devido à maior tenacidade à fratura, maior resistência ao impacto e maior tolerância a danos em relação aos termorrígidos. Devido à natureza reciclável e reformável, compósitos termoplásticos são selecionados para aplicações ambientalmente sustentáveis. Desta forma, estes apresentam uma ampla gama de aplicações em transporte terrestre, aeroespacial, e estruturas marinhas [ $[3,4, \underline{5}]$.

O Poli (éter-imida) é um termoplástico de engenharia, sendo este um polímero amorfo, transparente e de coloração amarelada, apresentando elevada temperatura de transição vítrea (aproximadamente $217^{\circ} \mathrm{C}$ ), caracterizado por elevada resistência mecânica e rigidez, tanto à temperatura ambiente quanto a temperaturas superiores. Sua estrutura química consiste em repetidas unidades de imidas aromáticas e éter. Como principais vantagens em relação a sua estrutura encontram-se: boas resistência e rigidez em elevadas temperaturas; alta resistência térmica; boas propriedades elétricas e ampla resistência química. Diferente de outros polímeros de engenharia, o PEI pode ser facilmente amolecido e conformado em alta escala [풍] .

Os compósitos com matriz polimérica PEI reforçados com fibras de carbono e de vidro, conhecidos também como híbridos, são obtidos utilizando-se dois ou mais tipos de fibras diferentes e uma única matriz. Estes compósitos híbridos são mais resistentes e mais tenazes, possuindo maior resistência ao impacto e podem ser produzidos em menor custo do que com os respectivos compósitos totalmente reforçados com fibras de carbono ou com fibras de vidro [ㄱ, $\underline{8}, \underline{9}]$. Além disso, a existência de uma camada de fibra de vidro promove isolamento elétrico nesta região.

Soldagem por resistência elétrica é uma das técnicas de soldagem mais atraentes para termoplásticos hoje em dia. É um método bastante simples, que utiliza elementos eletricamente resistivos 
a passagem de corrente elétrica, unidos entre as superfícies de ligação dos laminados ou compósitos para proporcionar o calor necessário para a junção. Uma corrente elétrica é aplicada ao elemento denominado resistivo ou de aquecimento para o aumento de sua temperatura, devido ao aquecimento por conta da resistividade elétrica deste material via efeito Joule. A temperatura do elemento resistivo aumenta consideravelmente na região de interface durante este processo, e a partir do efeito Joule, funde o polímero termoplástico diretamente em contato com o elemento resistivo. Quando a passagem de corrente é interrompida ocorre à união sob a aplicação de pressão a partir do resfriamento e solidificação da matriz polimérica, sendo que o elemento de aquecimento permanece na junta. A Figura 2 ilustra de maneira simplificada este processo de soldagem de compósitos $[\underline{10}, \underline{11}, \underline{12}]$.

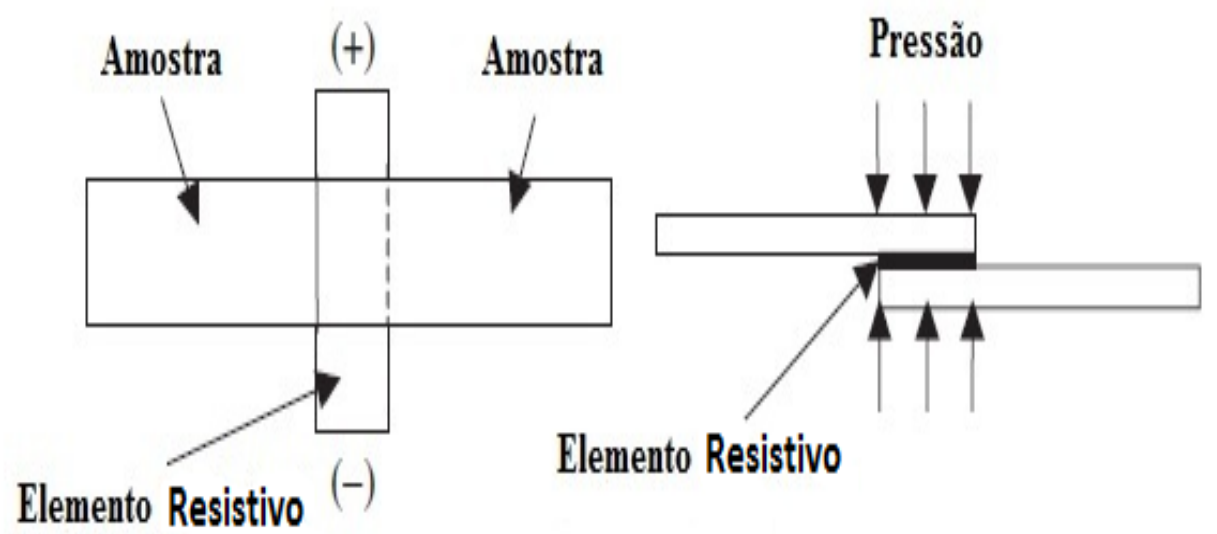

Figura 2: Diagrama esquemático do processo de soldagem por resistência em compósitos.

A soldagem por resistência elétrica tem um grande potencial para a integração de compósitos termoplásticos de alto desempenho, especialmente quando se trata de aplicações aeroespaciais. Este processo requer utilização de ferramentas muito simples e pouco ou nenhum tratamento de superfície dos materiais. As desvantagens desta técnica de soldagem são a possibilidade de movimento das fibras nos compósitos e problemas com aquecimentos irregulares. Entre estas desvantagens e devido às muitas dificuldades ainda presentes no processo, esta ainda não é considerada como uma técnica de junção principal de compósitos. Neste sentido, parâmetros como isolamento térmico, energia de entrada (kJ), tempo de soldagem, orientação da fibra, e o tipo de elemento resistivo a ser utilizado estão sendo estudados para melhoria da qualidade e desempenho das juntas soldadas $[\underline{13}, \underline{14}, \underline{15}, \underline{16}]$.

Há diversos caminhos para se atingir o objetivo da melhoria de um processo com a utilização da estatística, mais precisamente, pode-se realizá-lo a partir de um planejamento experimental. Entretanto, antes de definir o procedimento experimental mais adequado é necessária uma análise profunda do processo, buscando-se todas as informações possíveis na literatura, para que se leve em conta todas as variáveis a serem estudadas, as faixas de estudo para cada uma destas e as respostas de interesse. Em casos que existem vários fatores de interesse eu um determinado experimento, um planejamento fatorial deve ser utilizado. A resolução de um problema por meio de um planejamento estatístico conhecido como o planejamento experimental fatorial é utilizado para solução de um projeto experimental, usando de um número menor de medidas, mas que ao mesmo tempo consegue-se explorar todo o espaço experimental de interesse. Relacionado a trabalhar-se com 2 ou 3 variáveis independentes ou fatores, recomenda-se um delineamento composto central rotacional (DCCR) como ilustrado na Figura $3[\underline{17, \underline{18}] .}$. 


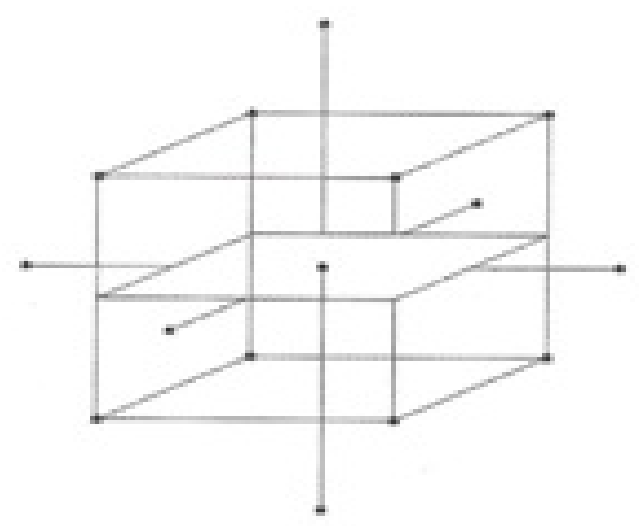

Figura 3: Representação de condução de experimentos com três variáveis independentes por delineamento composto central rotacional (DCCR) [17].

Os efeitos calculados em um fatorial $2^{3}$ podem ser interpretados geometricamente como um cubo em que os oito ensaios da matriz do planejamento correspondem aos seus vértices. Com esta ferramenta estatística ainda é possível à elaboração de um modelo matemático que validado estatisticamente pode ser utilizado para obtenção de superfície de resposta e assim poder determinar as condições otimizadas, conhecendo-se as significâncias estatísticas das respostas $[\underline{19}, \underline{20}]$. Neste sentido, neste trabalho foi utilizada esta ferramenta para avaliação de valores otimizados de corrente elétrica, tempo e pressão no processo de soldagem por resistência elétrica do compósito híbrido de PEI.

\section{MATERIAIS E MÉTODOS}

\subsection{Laminados}

Para a realização deste trabalho foram utilizados laminados de PEI reforçados com fibras de carbono e vidro, denominado como laminado PEI/híbrido. Estes laminados foram fornecidos pela empresa holandesa TenCate Advanced Composites. Este material foi confeccionado com tecido na configuração 8 HS com espessuras nominais entre 2 a 3,5mm, e com configuração (0/90)5s, contendo aproximadamente $50 \%$ em volume de matriz.

\subsection{Elementos Resistivos}

Como elementos resistivos para os testes de soldagem com os compósitos foram utilizadas malhas metálicas de aço inox AISI 304 de 200 mesh. As malhas, em forma de telas, foram adquiridas comercialmente da empresa SOLOTEST. As principais características técnicas da malha metálica são apresentadas na Tabela 1.

Tabela 1: Características da malha metálica apresentadas pelo fornecedor.

\begin{tabular}{c|c|c|c}
\hline Material & Mesh & $\begin{array}{c}\text { Diâmetro } \\
\text { do fio } \\
\text { (mm) }\end{array}$ & $\begin{array}{c}\text { Abertura } \\
\text { (mm) }\end{array}$ \\
\hline AISI 304 & 200 & 0,058 & 0,070 \\
\hline
\end{tabular}

\subsection{Processos de Soldagem}

Para o desenvolvimento deste trabalho, foi utilizada uma máquina de soldagem por resistência elétrica para compósitos, projetada e construída pela empresa AUMEK que consiste basicamente em uma máquina de prensagem com conectores elétricos integrados, para garantir aquecimento e pressão local durante o processo de soldagem. A Figura 4 apresenta uma ilustração da máquina de soldagem utilizada. 


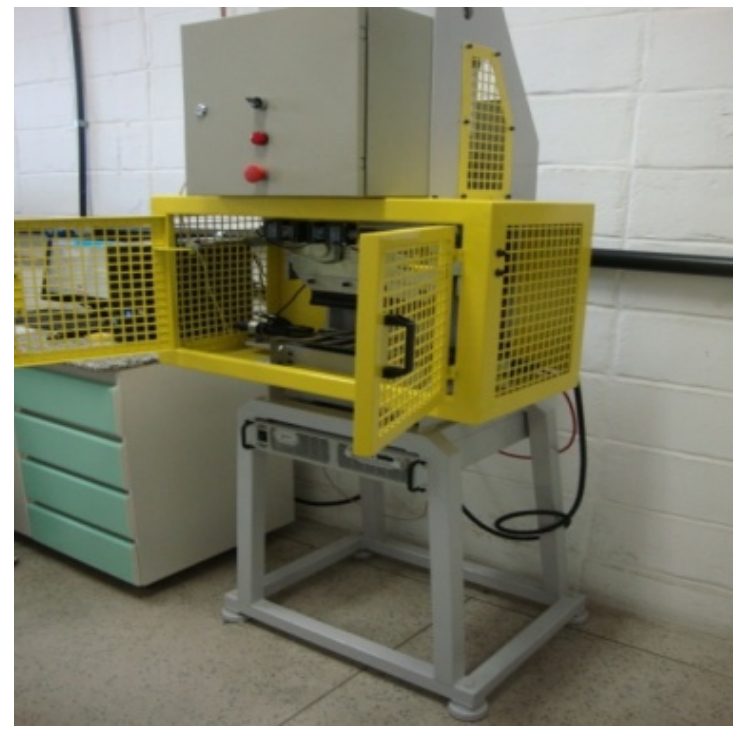

Figura 4: Foto da máquina de soldagem por resistência elétrica utilizada.

\subsection{Microscopia Ótica}

Para caracterização dos laminados e avaliação de eficiência do processo de soldagem por resistência, serão realizadas análises por microscopia óptica utilizando Microscópio Lupa Zeiss/Stemi 2000. Para as análises de microscopia ótica, os materiais utilizados foram um microscópio óptico com aumento de 50x a 1000x do fabricante Olympus, estereoscópico com aumento de 6,7x a 45x, maquina de corte cut-off com lubrificação (refrigerado) AROTEC, discos abrasivos para corte de materiais duros e moles, lixadeira circular e politriz circular do fabricante arotec, pistas de lixamento manual, lixas d’água de granulação 180, 220, 320, 400, 600, 800, 1000 e 1200 mesh, panos de polimento em pasta de diamante e alumina, alumina de $1 \mu \mathrm{m}$ e pasta de diamante de $6 \mu \mathrm{m}$ para polimento.

\subsection{Teste lap shear}

O teste de lap shear é o método mais utilizado para investigar a resistência à ruptura de juntas soldadas a partir do processo de resistência elétrica. Neste trabalho, este método foi utilizado para determinação da variável resposta do planejamento experimental. O método consiste em aplicação de força de tração até a ruptura em corpos de prova soldados medindo-se a tensão de ruptura da junta, sendo que neste trabalho utilizou-se juntas simples. Os ensaios foram realizados em uma máquina universal de ensaios da SHIMATZU baseando-se na norma ASTM D1002-10. As amostras foram ensaiadas a velocidade de 1,5 mm / min. (0,05 in / min.) até a falha sendo computado o valor máximo obtido. Os corpos de provas preparados para este ensaio são ilustrados na Figura 5 e o ensaio da amostra ilustrado pela Figura 6.

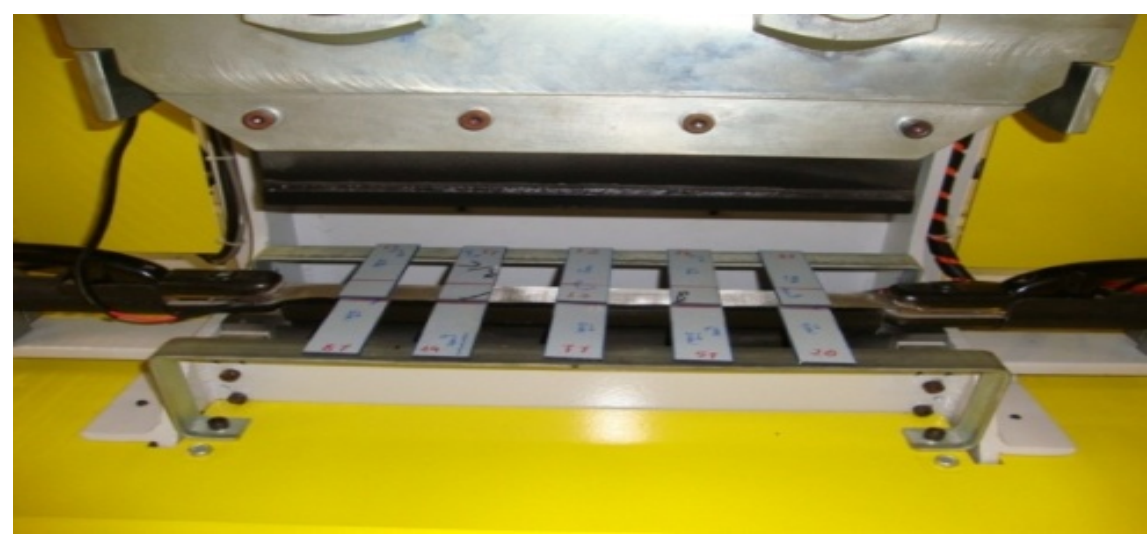

Figura 5: Corpos de Prova preparados para os ensaios de lap shear para avaliação dos melhores parâmetros de soldagem. 


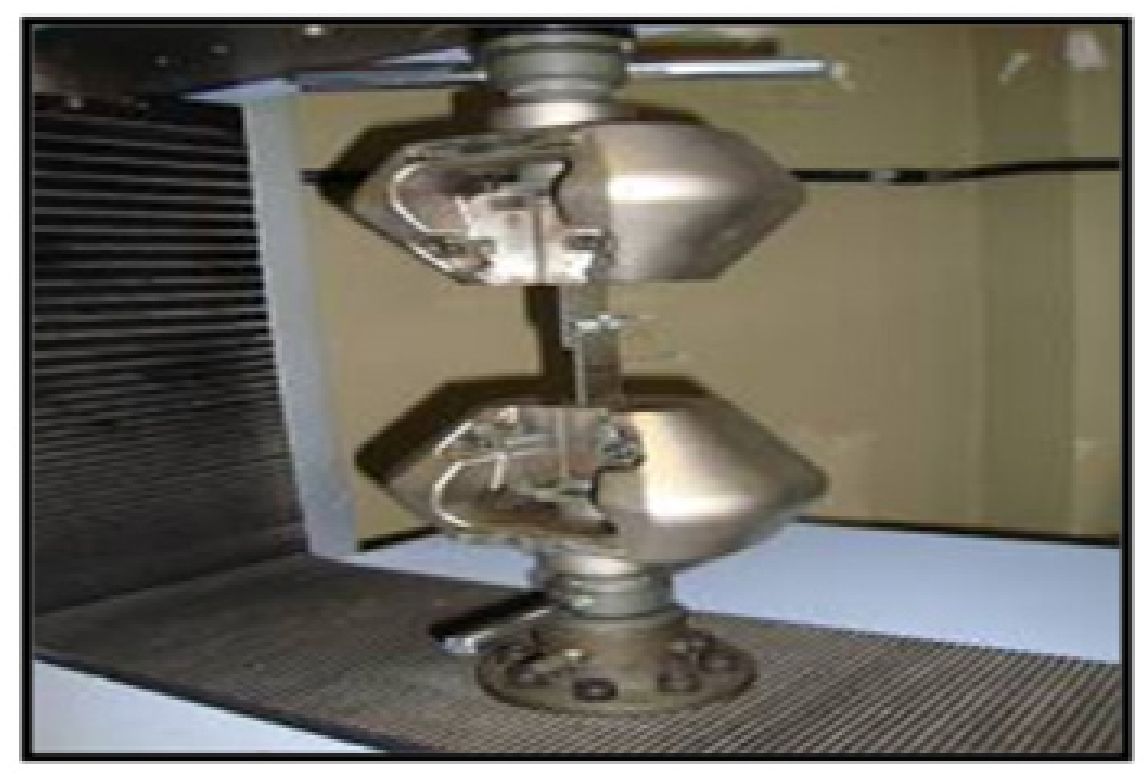

Figura 6: Detalhe da amostra no momento da falha durante o ensaio de lap shear.

\subsection{Planejamento Experimental}

Para determinação dos melhores parâmetros para o processo de soldagem por corrente elétrica, foi utilizado o planejamento experimental fatorial completo $2^{3}$, do tipo estrela rotacional com 6 replicatas no ponto central. Este planejamento permite os três efeitos das variáveis sobre as respostas estudadas, sendo estas estimadas junto com as interações das variáveis e também quando se deseja avaliar duas ou três variáveis ou fatores independentes em um processo ou experimento [토].

Os resultados obtidos foram analisados com auxílio dos softwares Estatística versão 5 (StatSoft Inc., US) e Design-Expert 6.0 (Stat-Ease Corporation, USA). O planejamento experimental foi realizado considerando como variáveis: pressão, corrente elétrica e tempo no processo; como resposta foi realizada o ensaio lap shear. Na Tabela 2 são apresentados os níveis reais e codificados para as variáveis tempo, corrente elétrica e pressão, sendo que estes valores máximos, mínimos e médios (+1, -1 e 0) foram estipulados com base em trabalho anterior do grupo de pesquisa [20]. Os valores de $\alpha$ calculados conforme a Equação 1.

$$
\alpha=(2 k)^{\frac{1}{4}}
$$

Em que: $\alpha=$ pontos axiais sobre o centro de cada face do espaço fatorial, ou seja, $\alpha=+1$ ou $\alpha=-$ $1 ; \mathrm{k}=\mathrm{o}$ número de variáveis do processo.

Tabela 2: Níveis reais e codificados para as variáveis: tempo, corrente elétrica e pressão, avaliados segundo planejamento experimental estrela rotacional para o compósito PEI/ fibra de vidro e carbono (híbrido).

\begin{tabular}{c|c|c|c|c|c|c}
\hline \multicolumn{2}{c|}{} & \multicolumn{5}{c}{ Níveis } \\
\hline Reais & Codificadas & $-\boldsymbol{\alpha}$ & $\mathbf{- 1}$ & $\mathbf{0}$ & $\mathbf{1}$ & $+\boldsymbol{\alpha}$ \\
\hline Tempo (s) & X1 & 30 & 50 & 175 & 300 & 350 \\
\hline Corrente (A) & X2 & 27,0 & 31,0 & 31,5 & 33,0 & 36,0 \\
\hline Pressão (MPa) & X3 & 0,70 & 0,70 & 1,85 & 3,00 & 3,00 \\
\hline
\end{tabular}




\section{RESULTADOS}

\subsection{Planejamento Experimental}

Os resultados dos experimentos realizados com os resultados adquiridos da variável resposta são apresentados na Tabela 3.

Tabela 3: Valores das variáveis estudadas e resposta correspondente aos experimentos conduzidos de acordo com o planejamento estatístico para o compósito PEI/ híbrido

\begin{tabular}{|c|c|c|c|c|c|c|c|}
\hline \multirow{3}{*}{ Experimento } & \multicolumn{6}{|c|}{ Variáveis Estudadas } & \multirow{3}{*}{$\begin{array}{c}\text { Variável } \\
\text { Resposta } \\
\text { Tensão de } \\
\text { cisalhamento } \\
\text { (MPa) }\end{array}$} \\
\hline & \multicolumn{3}{|c|}{ Codificadas } & \multicolumn{3}{|c|}{ Reais } & \\
\hline & Tempo (s) & Corrente (A) & $\begin{array}{c}\text { Pressão } \\
\text { (MPa) }\end{array}$ & $\begin{array}{c}\text { Tempo } \\
\text { (s) }\end{array}$ & $\begin{array}{c}\text { Corrente } \\
\text { (A) }\end{array}$ & $\begin{array}{c}\text { Pressão } \\
\text { (MPa) }\end{array}$ & \\
\hline 1 & -1 & -1 & -1 & 50 & 25 & 0,70 & 19,65 \\
\hline 2 & 1 & -1 & -1 & 300 & 25 & 0,70 & 11,33 \\
\hline 3 & -1 & -1 & -1 & 50 & 30 & 0,7 & 20,26 \\
\hline 4 & 1 & -1 & -1 & 300 & 30 & 0,7 & 4,11 \\
\hline 5 & -1 & 1 & 1 & 50 & 25 & 3,00 & 14,12 \\
\hline 6 & 1 & 1 & 1 & 300 & 25 & 3,00 & 11,80 \\
\hline 7 & -1 & 1 & 1 & 50 & 30 & 3,00 & 19,74 \\
\hline 8 & 1 & 1 & 1 & 300 & 30 & 3,00 & 9,75 \\
\hline 9 & -1 & 0 & 0 & 50 & 27,50 & 1,85 & 10,70 \\
\hline 10 & 3 & 0 & 0 & 300 & 27,50 & 1,85 & 10,00 \\
\hline 11 & 0 & 0 & 0 & 175 & 23,30 & 1,85 & 17,61 \\
\hline 12 & 0 & 0 & 0 & 175 & 31,70 & 1,85 & 3,95 \\
\hline 13 & 0 & -1 & -1 & 175 & 27,50 & 0,70 & 15,20 \\
\hline 14 & 0 & 1 & 1 & 175 & 27,50 & 3,00 & 12,03 \\
\hline 15 & 0 & 0 & 0 & 50 & 25 & 0,7 & 9,00 \\
\hline 16 & 0 & 0 & 0 & 50 & 25 & 0,7 & 10,00 \\
\hline 17 & 0 & 0 & 0 & 50 & 25 & 0,7 & 8,00 \\
\hline 18 & 0 & 0 & 0 & 50 & 25 & 0,7 & 7,50 \\
\hline 19 & 0 & 0 & 0 & 50 & 25 & 0,7 & 7,50 \\
\hline
\end{tabular}

Neste estudo os valores de tensão de cisalhamento por lap shear na soldagem do compósito híbrido se apresentou na faixa de 3,95 a 20,26 MPa, sendo o valor máximo observado para a combinação realizada no experimento 3 (Tabela 3). Esta diferença de faixa de valores para a variável resposta foi devido à escolha dos parâmetros para o planejamento experimental de modo que utilizasse de soldagem nas condições mínimas de tempo e corrente, explorando um espaço amostral grande para elaboração de um modelo estatístico adequado para otimização do processo. 


\subsection{Microscopia da junta soldada}

Para verificação da qualidade das juntas soldadas nas melhores condições selecionadas pelo planejamento experimental foi realizada a microscopia óptica. As micrografias adquiridas dos laminados PEI/Hibrido são mostrados na Figura 7 e pela Figura 8.

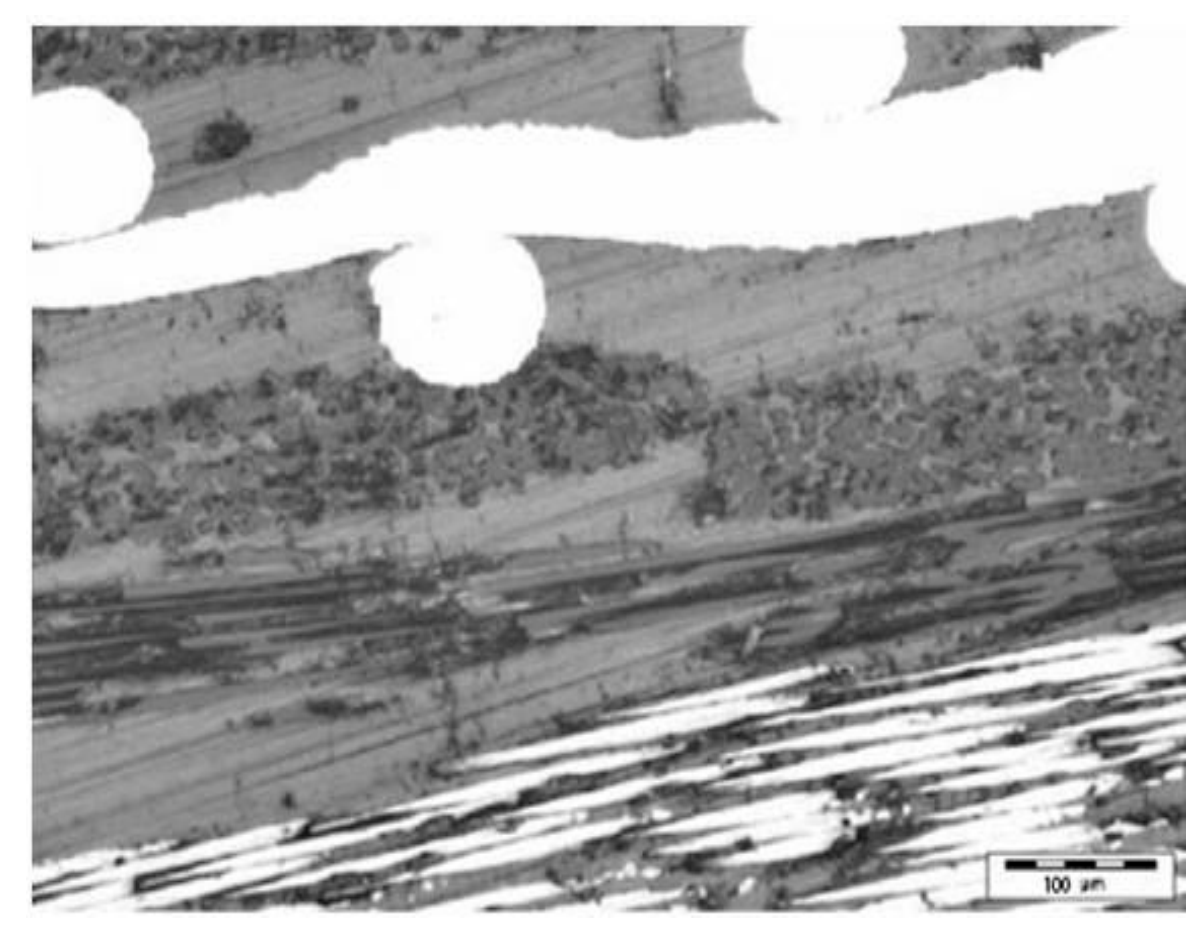

Figura 7: Micrografia óptica da região soldada do laminado do PEI/Híbrido nas melhores condições estudadas, 200X.

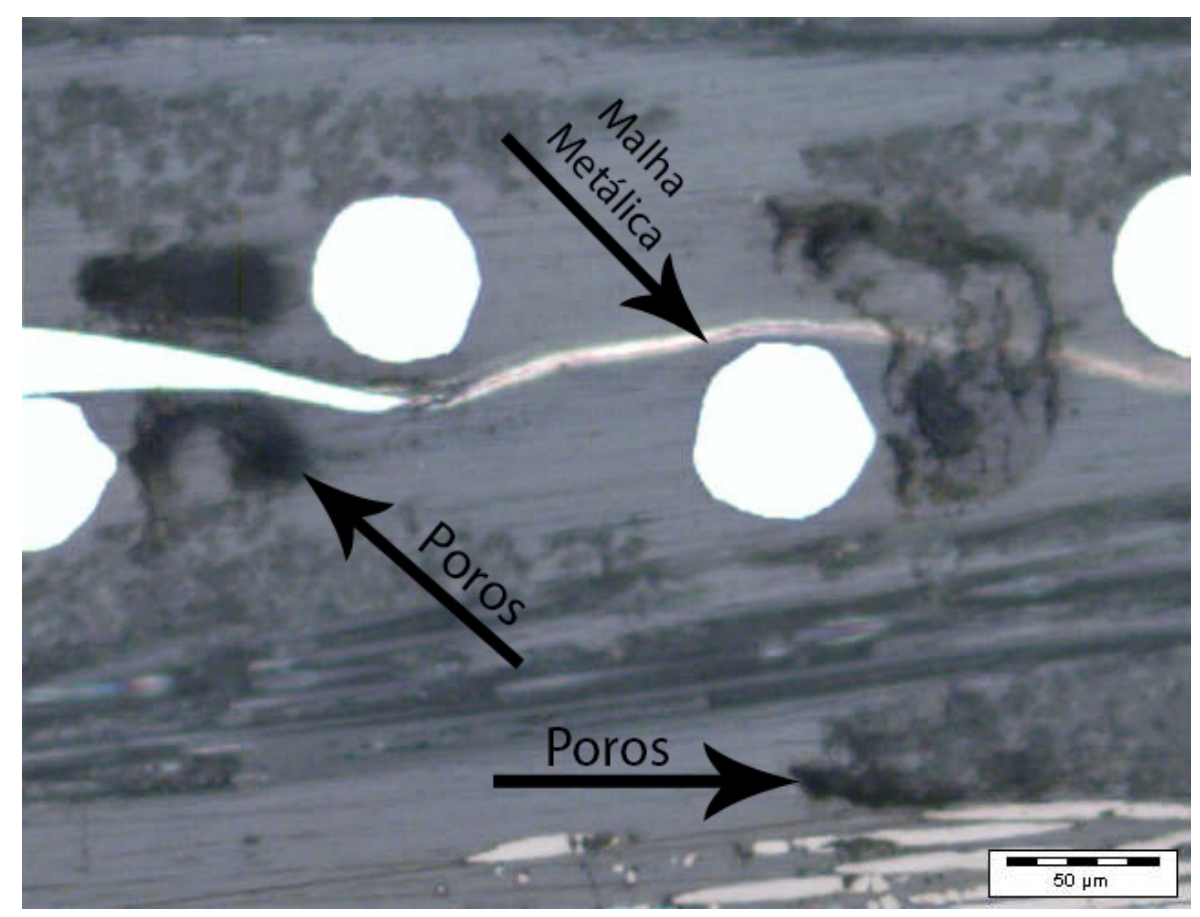

Figura 8: Micrografia óptica da região soldada do laminado PEI/Hibrido nas melhores condições estudadas, 500X. 


\subsection{Análise da junta após lap shear}

Foram realizadas as imagens da junta visualmente e por meio de microscopia ótica para verificação do aspecto dos materiais após ensaio mecânico de lap shear como ilustrado na Figura 8. Adicionalmente foi feita uma fotografia ampliada da região fraturada para verificação do tipo de fratura que se deve ao comportamento do elemento resistivo e a parte polimérica superficial do compósito, ilustrado na Figura 9 e também a microscopia ótica com o intuito de verificação do contato entre a malha e o polímero depois de solidificado, Figura 10.

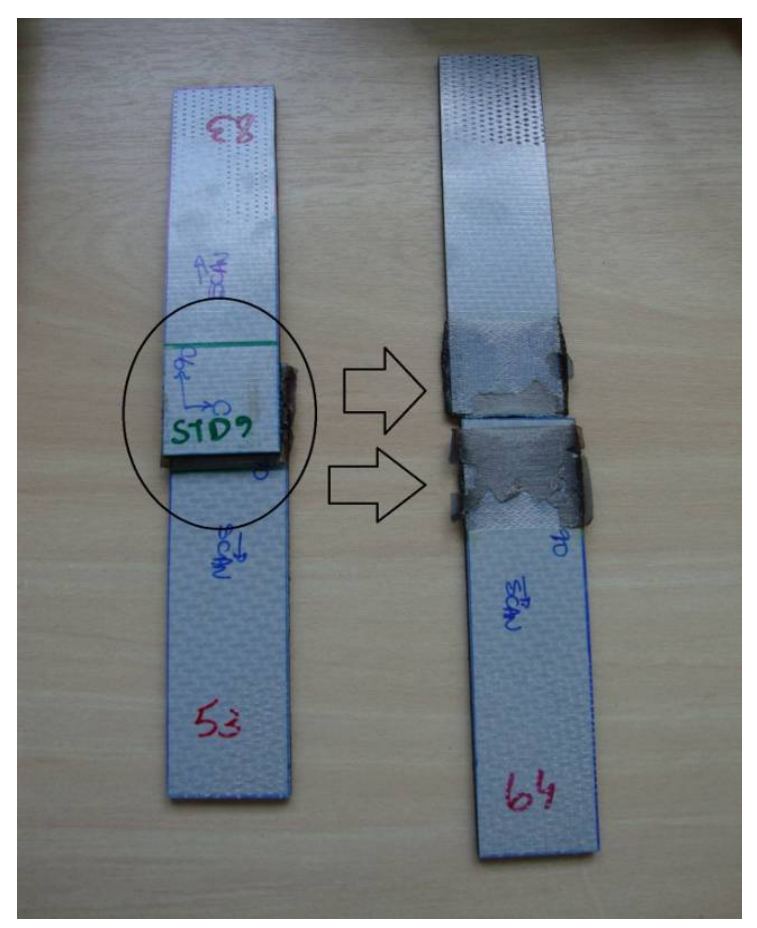

Figura 9: Fotografia da junta soldada antes e após o ensaio mecânico de lap shear.

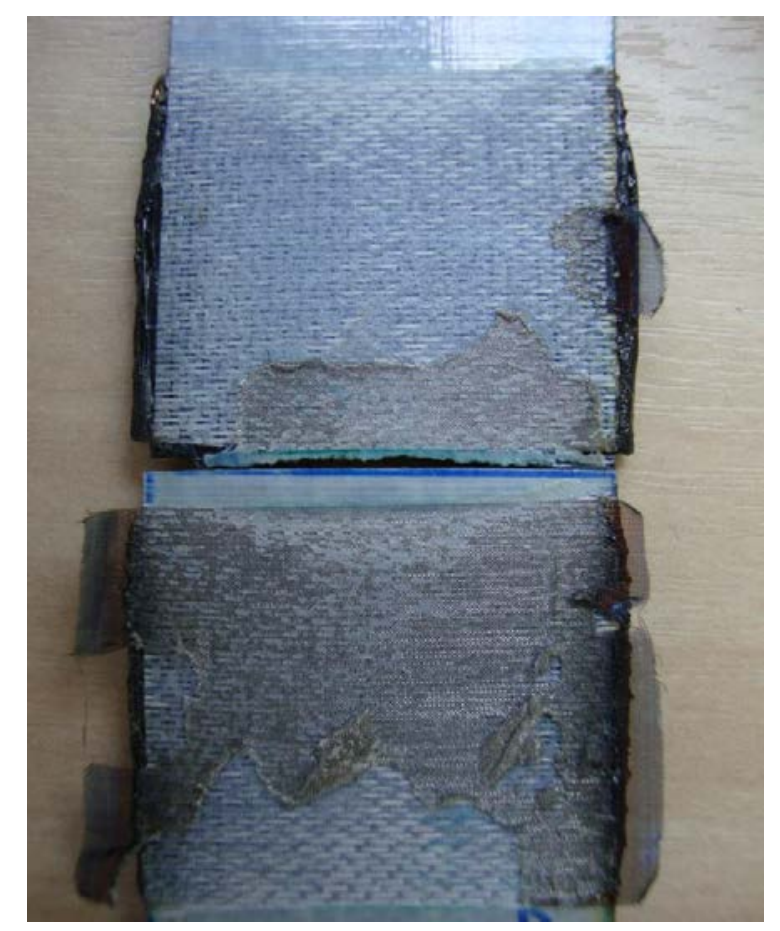

Figura 10: Fotografia ampliada da região fraturada após o ensaio mecânico de lap shear.

Foi realizada a microscopia ótica da região fraturada do ensaio de lap shear, em que o objetivo é 
melhor visualizar as regiões e o tipo de fratura. Foi feita a imagem da região fraturada em estereoscópio com ampliação de 20 vezes como ilustra a Figura 11.

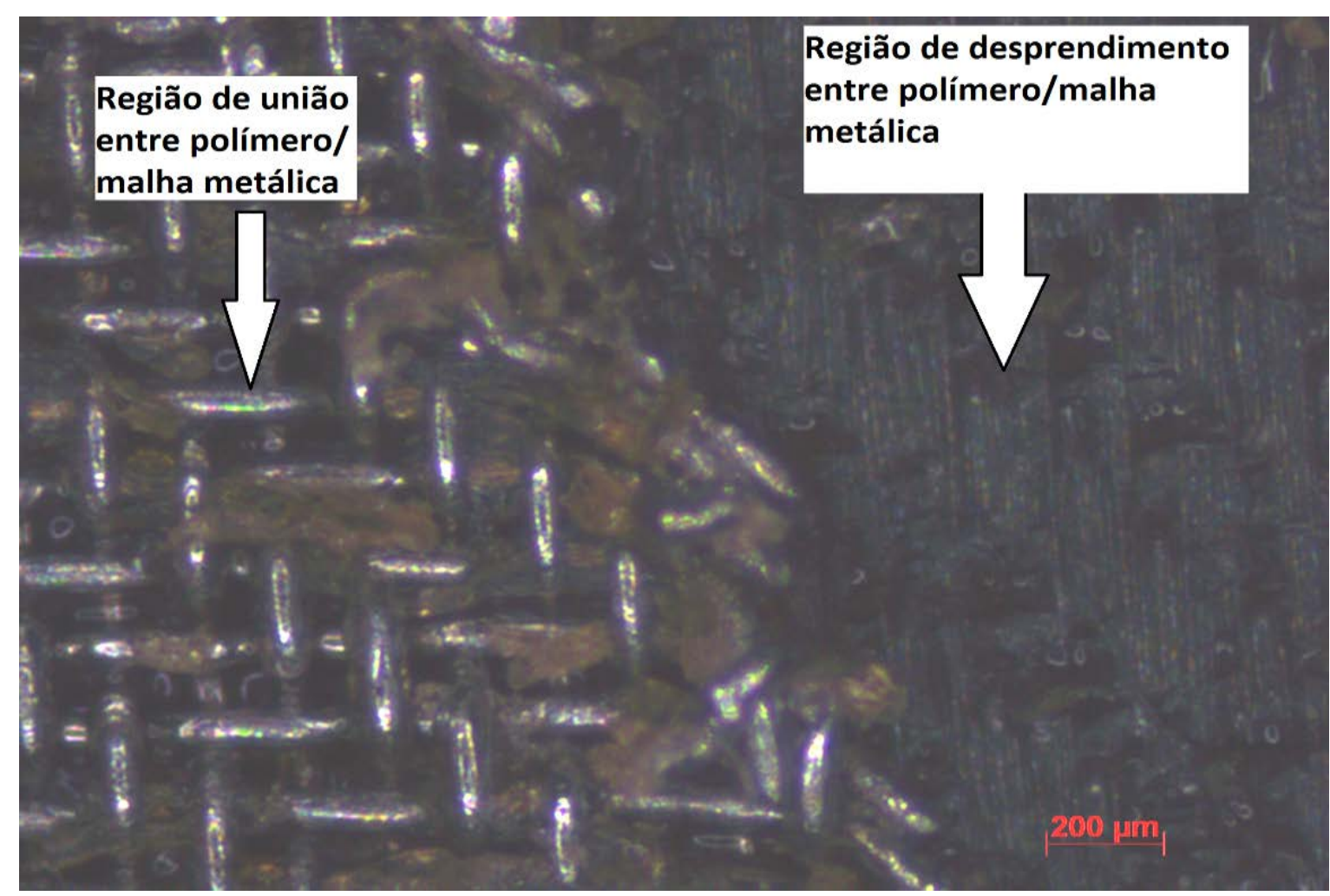

Figura 11: Imagem da região fraturada de lap shear com ampliação de 20X.

\section{DISCUSSÃO}

\subsection{Análise estatística do planejamento experimental}

Com o intuito de assegurar a confiabilidade na análise estatística realizada anteriormente, neste trabalho optou-se também pela realização da análise de variância. A análise de variância é um procedimento estatístico, que se refere à soma dos quadrados calculados a partir das informações retiradas do processo. Esta é utilizada para medir o efeito individual dos fatores [21].

De posse da construção do modelo, a significância do mesmo é verificada por meio da Análise de Variância (ANOVA), sendo que este teste avalia se o modelo é estatisticamente significativo. Pelo ANOVA também é possível verificar quais são os termos significativos do modelo e quais podem ser removidos. Os modelos são ajustados por meio do coeficiente de determinação (R2), que representa o percentual de variação na resposta explicada pelo modelo construído. Associado com este coeficiente tem-se o $R^{2}$ ajustado ( $R^{2}\left(\right.$ adj.)), que leva em consideração o fato de que o $R^{2}$ tende a superestimar a quantidade atual de variação contabilizada para a população. O teste p e F trata de métodos estatísticos que estimam dentro do intervalo de confiança do modelo o grau de validação e determinam os efeitos significativos do processo em estudo. [19]. Os resultados da ANOVA para os estudos dos efeitos das variáveis, tempo, pressão e corrente elétrica para a soldagem dos compósitos de PEI/ híbrido são apresentados na Tabela 4. 
Tabela 4: Análise de variância (ANOVA) para o modelo quadrático da soldagem por resistência elétrica do compósito PEI/híbrido nos experimentos conduzidos de acordo com o planejamento estatístico.

\begin{tabular}{|c|c|c|c|c|c|}
\hline Fator & $\begin{array}{c}\text { Soma } \\
\text { Quadrática }\end{array}$ & $\begin{array}{l}\text { Graus de } \\
\text { liberdade }\end{array}$ & $\begin{array}{c}\text { Média } \\
\text { quadrática }\end{array}$ & $\mathbf{F}$ & p \\
\hline Modelo & 372,61 & 9 & 41,40 & 5,90 & $0,0072 *$ \\
\hline $\mathrm{X}_{1}$ & 146,65 & 1 & 146,65 & 20,89 & $0,0013 *$ \\
\hline $\mathrm{X}_{2}$ & 74,56 & 1 & 74,56 & 10,62 & $0,0099 *$ \\
\hline $\mathrm{X}_{3}$ & 0,96 & 1 & 0,96 & 0,14 & 0,7198 \\
\hline $\mathrm{X}_{1}{ }^{2}$ & 74,48 & 1 & 74,48 & 10,61 & $0,0099 *$ \\
\hline $\mathrm{X}_{2}{ }^{2}$ & 8,62 & 1 & 8,62 & 1,23 & 0,2965 \\
\hline $\mathrm{X}_{3}{ }^{2}$ & 79,23 & 1 & 79,23 & 11,29 & 0,0084 \\
\hline $\mathrm{X}_{1} \mathrm{X}_{2}$ & 29,99 & 1 & 29,99 & 4,27 & 0,0687 \\
\hline $\mathrm{X}_{1} \mathrm{X}_{3}$ & 18,45 & 1 & 18,45 & 2,63 & 0,1394 \\
\hline $\mathrm{X}_{2} \mathrm{X}_{3}$ & 12,98 & 1 & 12,98 & 1,85 & 0,2070 \\
\hline Falta de ajuste & 48,87 & 5 & 9,77 & 2,73 & 0,1757 \\
\hline Erro residual & 63,17 & 9 & 7,02 & & \\
\hline Erro Puro & 14,30 & 4 & 3,58 & & \\
\hline$\overline{\mathbf{R}^{2}}$ & 0,7101 & & & & \\
\hline
\end{tabular}

${ }^{*} p$ : Valores significativos para valores de $p<0,05, \mathrm{X}_{1}$ : tempo codificado; $\mathrm{X}_{2}$ : corrente codificada; $\mathrm{X}_{3}$ : pressão codificada.

O modelo proposto para a análise da soldagem do laminado PEI/híbrido foi estatisticamente significativo sem falta de ajuste ao nível de confiança de $95 \%$, e mais de $71 \%$ da variabilidade experimental podendo ser explicada pelo modelo $\left(\mathrm{R}^{2}=0,7101\right)$. Em relação às significâncias das variáveis independentes estudadas neste trabalho, foram significativas, de acordo com a análise de variância, o tempo, a corrente elétrica, o tempo quadrático e a pressão quadrática.

O teste P (Tabela 4) indicou significância estatística ao nível de 99\% de confiança, não sendo constatada falta de ajuste para nenhum dos modelos avaliados (nível de confiança de 90\%). Verifica-se, ainda, a partir do valor de $\mathrm{R}^{2}$ que todas as equações ajustadas foram capazes de explicar mais de $70 \%$ da variabilidade dos valores experimentais. Desta forma, considerou-se que os modelos estatísticos obtidos foram adequados para descrever o processo de soldagem dos laminados em função do tempo, da corrente elétrica e pressão aplicada. A partir dos resultados das Tabelas de ANOVA, foi possível compor os modelos estatísticos incluindo os coeficientes correspondentes aos efeitos significativos para o processo de soldagem dos laminados estudados, sendo que os coeficientes relativos aos efeitos não significativos foram excluídos dos modelos. O modelo obtido por lap shear (MPa) é apresentado pela Equação 2.

$169,96+0,24 \cdot X_{1}-8,13 \cdot X_{2}-8,66 \cdot 10^{-5} \cdot X_{1}^{2}+3,20 \cdot X_{3}^{2}-0,01 \cdot X_{1} \cdot X_{2}+0,01 \cdot X_{1} \cdot X_{3}+0,73841 \cdot X_{2} \cdot X_{3}$

Em que: $X_{1}=$ Tempo de soldagem em segundos; $X_{2}=$ corrente aplicada em Amperes e $X_{3}=$ pressão aplicada nas peças na soldagem em MPa. 
A partir das condições otimizadas foram construídos utilizando-se o mesmo programa, gráficos de superfície de resposta referentes aos valores de variáveis buscando-se os maiores valores preditos pelo modelo dos ensaios de lap shear. A superfície de resposta com as regiões de otimização é apresentada na Figura 12, em que a pressão estipulada pelo programa foi de 0,7 Mpa.

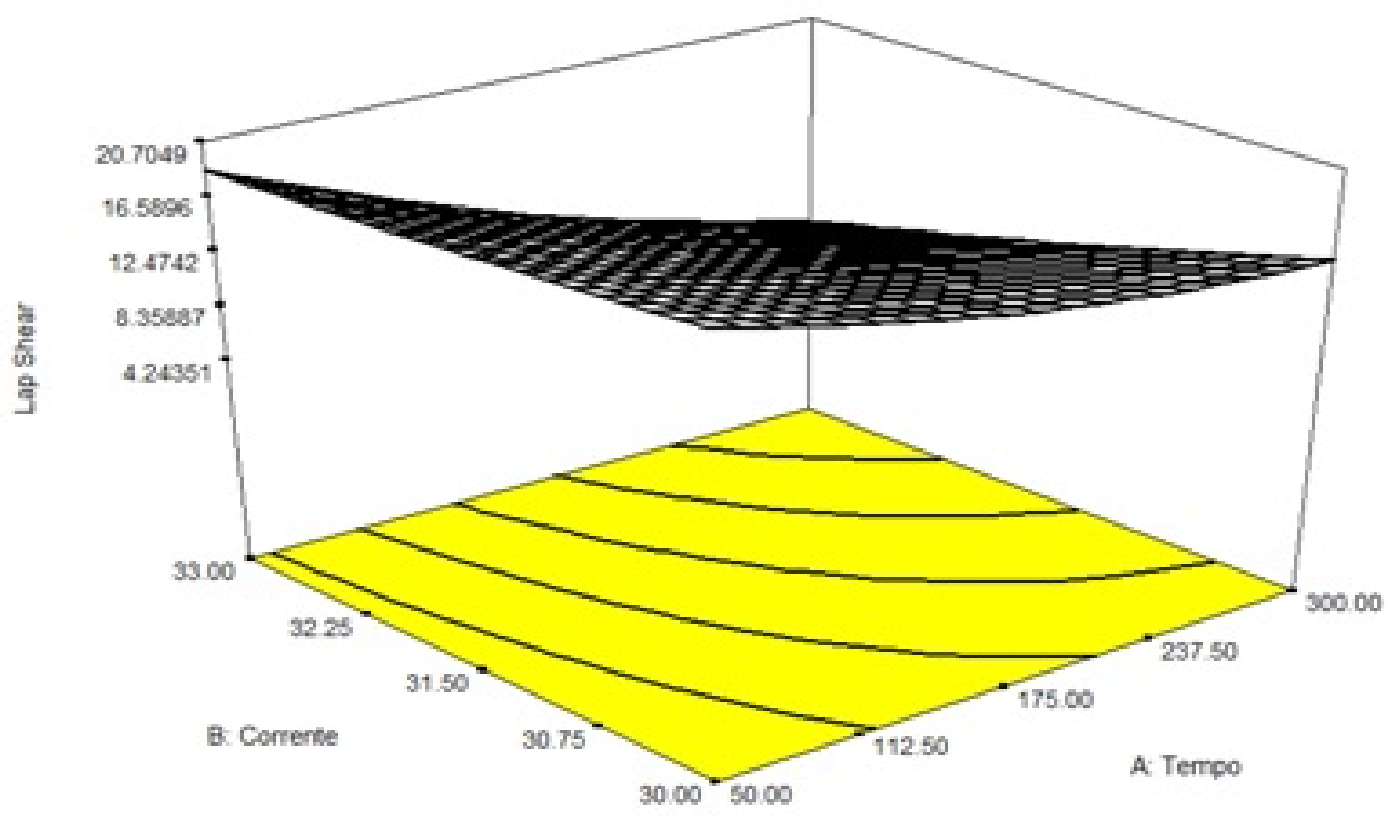

Figura 12: Superfície de resposta obtida pelo programa Design Express versão 6.0 dos parâmetros tempo e corrente em função do ensaio de lap shear, com a pressão de operação estipulada em 0,7 Mpa.

Com os resultados obtidos ainda foi possível construir pelo programa o gráfico da curva de nível referente à curva de superfície de resposta ilustrada na Figura 13.

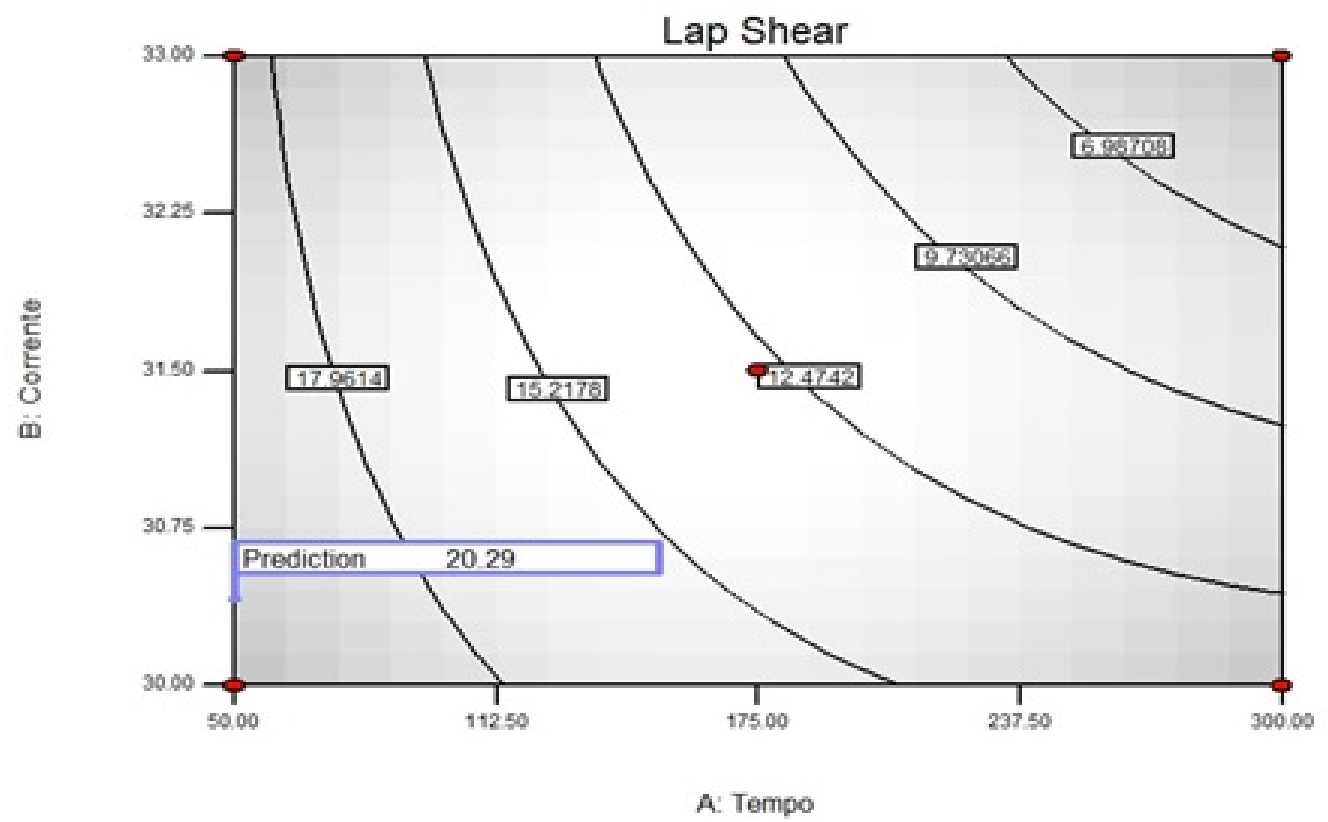

Figura 13: Curvas de nível para o laminado PEI/híbrido em função do tempo e da corrente elétrica aplicada obtida pelo programa estatístico.

A partir da análise da superfície de resposta e das curvas de nível apresentadas nas Figuras 10 e 11, podem ser verificados os valores das variáveis independentes significativas otimizadas pelo programa. 
Como se buscou valores da variável dependente máximos para a soldagem do compósito híbrido, os valores das variáveis independentes aprimoradas foram de 50,17s; 30,41 A e 0,7 MPa. Estes valores de parâmetros propostos pelo modelo estático predizem que para a junta soldada do laminado estudado um valor predito de lap shear de 20,29 Mpa, de acordo com o modelo proposto representado pela equação 2.

\subsection{Análise da qualidade da junta soldada}

\subsubsection{Microscopia Ótica das regiões após o ensaio de lap shear}

No material em estudado características de boa soldabilidade foram confirmadas pelas análises de microscopia ótica, ilustradas pelas Figuras 7 e 8, em que se verificou o recobrimento da malha metálica (elemento resistivo) e na solidificação do polímero foi possível constatar sua semelhança com o material polimérico sem ter passado pela fusão. Foi possível também observar a aparição de poros no material, oriundos do próprio laminado polimérico, certificando que a soldagem feita no material não foi prejudicial nas características morfológicas do compósito como evidenciado na Figura 8.

\subsubsection{Avaliação da falha após o ensaio de lap shear}

A observação visual do modo de falha de amostras testadas durante este ensaio também pode fornecer algumas informações importantes sobre a qualidade da solda. Três tipos de modos de falha de amostras são observados durante o ensaio de lap shear em compósitos sendo estes: intralaminar; interfacial e fratura da amostra, como apresentado esquematicamente na Figura 14.

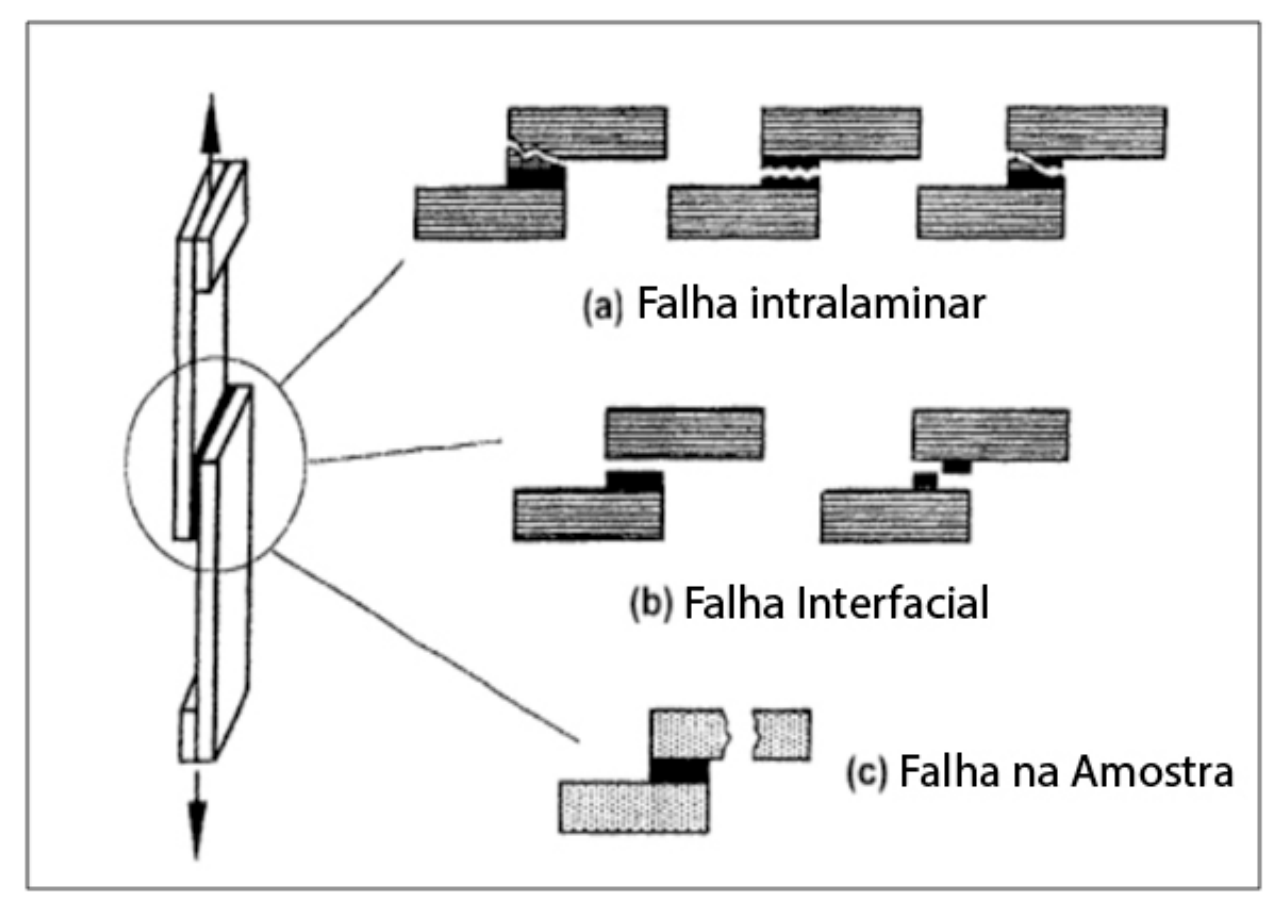

Figura 14: Modos de falha típicos observados durante o ensaio de lap shear em compósitos: intralaminar, interfacial e fratura [11].

O modo de falha intralaminar é considerado nestes casos, quando a fratura ocorre na estrutura com grandes quantidades de material, no elemento de aquecimento (rompimento do elemento de aquecimento) ou em ambos. Este tipo de falha geralmente é acompanhado de maiores valores de resistência do teste de lap shear, implicando, assim, melhor qualidade de solda. A falha interfacial ocorre na interface entre o laminado e o elemento de aquecimento ou resistivo e rendimentos mais baixos de valores da resistência mecânica observada no ensaio de lap shear, geralmente como parâmetro de resposta a baixa eficiência do processo de soldagem [11]. As juntas deste estudo que apresentaram valores de tensão de cisalhamento pelo ensaio de lap shear na faixa de 4 a 10 Mpa apresentaram o tipo de falha interfacial.

Como observado na Figura 10 e confirmado em análise da Figura 11, o tipo de falha obtido pelo 
ensaio de lap shear é do tipo intralaminar. Foram evidenciadas as duas regiões principais de fratura referente ao ensaio mecânico: a região da malha metálica em união com a matriz polimérica e a região polimérica fundida que foi solidificada durante o processamento de soldagem e desprendida da malha metálica após o ensaio mecânico (Figura 11). Estas observações corroboram com as imagens anteriores fotografadas e indicam boa união das partes de compósito soldadas devido ao tipo de fratura intralaminar.

\section{CONCLUSÕES}

A partir dos resultados obtidos neste trabalho, foi possível estabelecer um melhor modelo estatístico para avaliar os parâmetros mais adequados para a soldagem por resistência elétrica do compósito em estudo. Desta forma, foi possível estabelecer para o compósito PEI/hibrido os valores de parâmetros para soldagem para obtenção de um valor de máximo de tensão de cisalhamento pelo ensaio mecânico escolhido como variável reposta adotada para o planejamento experimental. Adicionalmente, foram obtidos valores otimizados propostos pelo programa Design Express versão 6.0, que indicou a tendência para a utilização de um tempo de soldagem de 50,17 segundos; corrente elétrica de 30,83 ampères e pressão de 0,98 MPa, para obtenção de um valor máximo de tensão de cisalhamento da junta de $21 \mathrm{MPa}$ quando avaliado por ensaio de lap shear.

\section{AGRADECIMENTOS}

Agradecemos aos órgãos financiadores deste trabalho: FAPESP e CAPES.

\section{BIBLIOGRAFIA}

[1] PANNEERSELVAM, K.., ARAVINDAN, S., HAQ, A. N. "Study on resistance welding of glass fiber reinforced thermoplastic composites”, Materials and Design, v. 41, pp. 453-459, 2012.

[2] Sebastian Freissinet. WORRIES ABOUT NEW COMPOSITE MADE AIRPLANE, http://www.1001crash.com/index-page-composite-lg-2.html. Acessado em abril de 2014.

[3] MIRABEL, C. R.; BOTELHO, E. C. “O Uso de compósitos estruturais na indústria aeroespacial”, Polímeros: Ciência e Tecnologia, v. 10, n. 2, pp. 4-10, 2000.

[4] MANO, E. B. Polímeros como materiais de engenharia. 5a Ed., São Paulo, Editora Blucher, 2010.

[5] MEDEIROS, R., TITA, V., NETO, S. V. "Método experimental de análise de junções metalcompósito para estruturas aeronáuticas”, Revista UNIFA, v. 23, n. 29, pp. 57-68, 2011.

[6] OLIVEIRA, G, H., GUIMARÃES, V. A., BOTELHO, E. C. "Influência da temperatura no desempenho mecânico de compósitos PEI/fibras de vidro”, Polímeros: Ciência e Tecnologia, v. 19, n. 4, pp. 305-312, 2009.

[7] CALLISTER, W. D. Jr. "Compósitos” In: Ciência e Engenharia de Materiais: uma Introdução, $8^{\mathrm{a}}$ Ed., capitulo 16, Rio de Janeiro, GEN/ LTC, 2012.

[8] CATSMAN, P. “Polyetherimide (PEI): substituting metal”, Kunststoffe, v. 95, n.10, pp.143-147, 2005.

[9] SOUZA, S. D. B. "Avaliação dos parâmetros de soldagem por resistência para compósitos de PPS/Fibras contínuas com aplicações aeronáuticas”, Tese de M. Sc., Universidade Estadual Paulista/UNESP, Faculdade de Engenharia de Guaratinguetá, SP, 2013.

[10] YOUSEFPOUR, A., HOJJATI M., IMMRIGEON, P. J. "Fusion bonding/welding of thermoplastic composites”, Journal of Thermoplastic Composite Materials, v. 17, pp. 303-341, 2004.

[11] STAVROV, D., BERSEE, H. E. N. "Resistance welding of thermoplastic composites: an overview", Composites: Part A: applied science and manufacturing, v. 36, n. 1, pp. 39-54, 2005.

[12] MATSEN, M. R., MICCARVILLE, D. A., STEPAN, M. M. "Resistance welding of thermoplastics in aerospace structure”. US 7,126,096, 2006.

[13] DUBÉ, M. Static and fatigue behaviour Of thermoplastic composite laminates joined by resistance welding, Tese D.Sc., McGill University, Montreal, Canada, 2007.

[14] AGEORGES, C. L., HOU, Y. E. "Advances in fusion bonding techniques for joining thermoplastic matrix composites: a review”, Composites Part A: Applied Science and Manufacturing, v. 32, pp. 839857, 2001. 
[15] NINO, G.F., AHMED, T.J., BERSEE, H.E.N., BEUKERS, A. “Thermal NDI of resistance welded composite structures”, Composites: Part B, v. 40, pp. 237-248, 2009.

[16] PANNEERSELVAM, K.., ARAVINDAN, S., HAQ, A.N. "Study on resistence welding of glass fiber reinforced thermoplastic composites”, Materials and Design, v. 41, pp. 453-459, 2012.

[17] RODRIGUES, M. I., IEMMA A. F. Planejamento de Experimentos e Otimização de Processos uma Estratégia Sequencial de Planejamentos, Casa do Pão, Campinas, 2005.

[18] NETO, B.N., SCARMINIO, I.S., BRUNS, R. E. Como Fazer Experimentos, $4^{a}$ Ed., Bookman, 2010.

[19] MENDES P. K. Otimização da Solda de Pontos por Resistência Elétrica na Liga de Alumínio 5052H32 através do Projeto e Análise de Experimentos (DOE), Dissertação M.Sc, Universidade Federal de Itajubá, Minas Gerais, MG, 2011.

[20] COSTA, A. P. Efeito do condicionamento ambiental em compósitos soldados de PPS/Fibras contínuas, Dissertação M.Sc, Universidade Estadual Paulista, Faculdade de Engenharia de Guaratinguetá, São Paulo, SP, 2011.

[21] BUTTON, S. T. Metodologia para planejamento experimental e análise de resultados, Tese de M.Sc., Universidade Estadual de Campinas, Faculdade de Engenharia Mecânica, São Paulo, 2005. 